\title{
CDK3 expression and its clinical significance in human nasopharyngeal carcinoma
}

\author{
LIANG WANG $^{1 *}$, HONG-YI HU ${ }^{2 *}$, YI-LING LIN ${ }^{2}$, ZHI-XIANG ZHAO ${ }^{1}$, LU TAN $^{1}$, \\ PENG YU ${ }^{2}$, HUI-JUAN WAN ${ }^{2}, \mathrm{ZHE} \mathrm{JIN}^{1}$ and DUO ZHENG ${ }^{1}$ \\ ${ }^{1}$ Department of Basic Medicine, School of Medicine, Shenzhen University, Shenzhen, Guangdong 518060; \\ ${ }^{2}$ Department of Otolaryngology, Peking University Shenzhen Hospital, Shenzhen, Guangdong 518036, P.R. China
}

Received August 13, 2013; Accepted March 5, 2014

DOI: $10.3892 / \mathrm{mmr} .2014 .2095$

\begin{abstract}
The aim of the current study was to investigate the expression of cyclin-dependent kinase 3 (CDK3) in human nasopharyngeal carcinoma (NPC) and to evaluate its association with the clinicopathological characteristics of patients with NPC. CDK3 expression was examined in three NPC cell lines and one nasopharyngeal epithelial cell line by western blot analysis and in 94 specimens of NPC and 40 specimens of inflamed nasopharyngeal tissue by immunohistochemistry staining. CDK3 was overexpressed in the three NPC cell lines, 5-8F, CNE1 and CNE2, compared with the NP-69 nasopharyngeal epithelial cell line, and was primarily expressed in the cytoplasm. The frequency of CDK3 expression was significantly higher in NPC specimens (67\%) compared with inflamed nasopharyngeal tissue specimens $(12.5 \% ; \mathrm{P}<0.001)$. CDK3 expression was associated with the degree of infiltration, lymph node metastasis and tumor node metastasis clinical staging, respectively, $(\mathrm{P}<0.001)$ in patients with NPC. These results revealed that the expression of $\mathrm{CDK} 3$ is associated with the progression of NPC, and may be a potential biomarker for prediction of the prognosis of patients with NPC.
\end{abstract}

\section{Introduction}

Nasopharyngeal carcinoma (NPC) is a cancer of head and neck squamous cells and commonly observed in Southeast Asia, including certain regions of South China, such as Guangdong, Guangxi, Hunan and Fujian provinces $(1,2)$. According to the International Agency for Research on Cancer, there were an estimated 84,000 incident cases of NPC and

Correspondence to: Dr Zhe Jin or Dr Duo Zheng, Department of Basic Medicine, School of Medicine, Shenzhen University, 3688 Nanhai Avenue, Shenzhen, Guangdong 518060, P.R. China

E-mail: zhejin1995@yahoo.com

E-mail: zhengduo1228@gmail.com

*Contributed equally

Key words: cyclin-dependent kinase 3, nasopharyngeal carcinoma, biomarker, prognosis
51,600 mortalities due to NPC in 2008, with a reported annual incidence of $30-80 / 10^{5}$ individuals in endemic regions (3). At present, the main treatment strategy for NPC is radiotherapy; however, patients with advanced disease tend to experience therapeutic failure and the 5 year overall survival rate is $\sim 52 \%$ (4). Since NPC exhibits highly aggressive behavior (5) with rapid progression to mortality (6), it is essential to gain an improved understanding of the molecular events underlying the development of these tumors further improve survival rates. Thus, it further investigations into the molecular mechanisms of NPC are required.

The development and progression of NPC involves uncontrolled and indefinite proliferation, which is partially caused by abnormal cell cycle regulation. The change in cell cycle distribution, as well as abnormal expression and activity of associated regulatory factors, including cell cycle protein (cyclin), cyclin-dependent kinase (CDK) and its inhibitor cyclin-dependent kinase inhibitor (CKI), may result in cell cycle disorder and abnormal cell proliferation. CDK3, a member of the CDK family, was originally classified as a CDK due to its high sequence identity (76\%) with CDC2 and CDK2 (7). CDK3 is critical in cell cycle regulation and is involved in G0-G1 and G1-S stage cell cycle transitions (8-13). Furthermore, CDK3 was overexpressed in a number of cancer cell lines, and may be important role in cell proliferation and malignant transformation (14-17).

CDK3 has been previously reported to be highly expressed in human glioblastoma (15). Ectopic expression of CDK3 enhances the transformation of JB6 Cl41 cells, and knockdown of endogenous CDK3 suppressed the proliferation and growth of T98G glioblastoma cells in soft agar (15), indicating that the CDK3 is important in tumorigenesis. However, the expression of CDK3 in NPC is unclear. The aim of the present study was to determine the association between CDK3 expression and the clinicopathological features of patients with NPC.

\section{Materials and methods}

Cell lines and tissue samples. CNE1, CNE2 and 5-8F NPC cell lines and the NP-69 nasopharyngeal epithelial cell line were provided by Professor Tie-Bang Kang (Sun Yat-Sen University Cancer Center, Guangzhou, China) and were cultured in Dulbecco's modified Eagle's medium (DMEM) with $10 \%$ 
fetal bovine serum (both from Invitrogen Life Technologies, Carlsbad, CA, USA) at $37^{\circ} \mathrm{C}$ in a $5 \% \mathrm{CO}_{2}$ incubator. A total of 134 specimens were provided by the Department of Otolaryngology, Beijing University Shenzhen Hospital (2010-2012), with signed consent forms. Among these, there were 94 cases of NPC (61 males and 33 females). The age of patients ranged between 18-78 years (median age, 44 years). There were a total of 40 cases of nasopharyngeal inflammation (20 males and 20 females), the age of patients ranged between 23-68 years (median age, 42.6 years). All specimens were examined by clinical and pathological diagnosis.

All specimens were fixed with $10 \%$ formalin, embedded in paraffin within three days and processed into $4-\mu \mathrm{m}$ sections. Rabbit polyclonal antibodies against CDK3 were purchased from Abcam (Cambridge, MA, USA). An Ultra Sensitive ${ }^{\mathrm{TM}} \mathrm{S}-\mathrm{P}$ Allergic kit (mouse/rabbit) and a DAB Color Development kit were purchased from Fuzhou Manxin Biological Technology Development, Inc. A Leica Application Suite Microscope (Leica Microsystems GmbH, Wetzlar, Germany) was used to capture images of the stained sections.

Western blotting. Cells were harvested at 80-90\% confluency, washed twice with cold phosphate-buffered saline (PBS) and lysed on ice in NP-40 cell lysis buffer $(50 \mathrm{mmol} / \mathrm{l}$ Tris- $\mathrm{HCl}$, $\mathrm{pH} 8.0,150 \mathrm{mmol} / \mathrm{l} \mathrm{NaCl}$ and $0.5 \% \mathrm{NP}-40$ ) with soybean trypsin inhibitor (Sigma, St. Louis, MO, USA). Protein concentration was measured using the detergent-compatible protein assay kit (Bio-Rad, Hercules, CA, USA) according to the manufacturer's instructions. Equal quantities of protein were separated electrophoretically on $10 \%$ sodium dodecyl sulfate (SDS)-polyacrylamide gels and transferred onto polyvinylidene difluoride membranes (Millipore, Bedford, MA, USA). The membrane was hybridized with the anti-CDK3 antibody (Abcam), and visualized using an enhanced chemiluminescence detection kit (Amersham Biosciences, Piscataway, NJ, USA). The membranes were stripped and reprobed with an anti-actin mouse monoclonal antibody (1:2,000 dilution; Millipore) as a loading control.

Immunohistochemistry (IHC). Hematoxylin and eosin staining was performed on tissue samples to identify histopathological properties and pathological classifications. Paraffin sections were dewaxed and hydrated, the nucleus was stained with hematoxylin and the cytoplasm was stained with $0.5 \%$ eosin. Following dehydration, sections were sealed with transparent and neutral gum. The staining results were carefully examined by experienced pathologists to determine the tumor node metastasis staging according to the Chinese clinical staging scheme of NPC in 2008 (18).

IHC was used to detect the expression of CDK3 in tissue samples. Paraffin sections were dewaxed and hydrated; sections were submerged in $0.01 \mathrm{mmol} / 1$ citrate buffer and microwaved for antigenic retrieval for $20 \mathrm{~min}$. Next, the antigens were cooled naturally, specimens were washed with PBS and incubated for $10 \mathrm{~min}$ at room temperature with endogenous peroxidase blockers (3\% hydrogen peroxide in methanol; Professional-Bio, Shenzhen, China). Following washing with PBS, normal non-immune animal serum (goat) was added and specimens were incubated for $10 \mathrm{~min}$ at room temperature. Specimens were washed with PBS and incubated for $60 \mathrm{~min}$ at

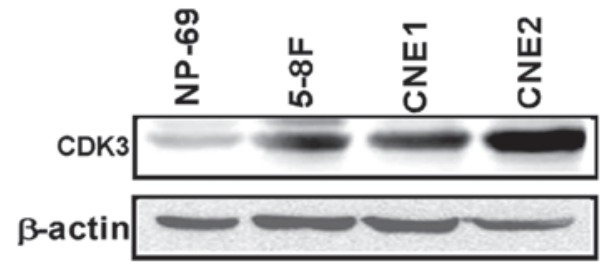

Figure 1. Expression of CDK3 was elevated in NPC cell lines. Expression analysis of CDK3 protein in the NP-69 nasopharyngeal epithelial cell line and NPC cell lines (5-8F, CNE1 and CNE2) by western blotting. Protein expression levels were normalized with $\beta$-actin. CDK3, cyclin-dependent kinase 3; NPC, nasopharyngeal carcinoma.

room temperature with $3 \mu \mathrm{g} / \mathrm{ml}$ primary antibody. Specimens were washed with PBS and incubated for $10 \mathrm{~min}$ at room temperature with the secondary, biotin-labeled antibody (goat anti-mouse IgG antibody; Abcam). Following washing with PBS, streptavidin-biotin immunoperoxidase solution (Abcam) was added and the specimens were incubated for $10 \mathrm{~min}$ at room temperature. Following washing with PBS, a color test was performed using the DAB Color Development kit (Zhongshan Golden Bridge, Beijing, China). The nucleus was stained with hematoxylin. Following dehydration, sections were sealed with transparent and neutral gum. PBS was used as a negative control instead of a primary or secondary antibody. The results were observed by microscopy. The cytoplasm or nuclei that were attached to brown or yellow particles were defined as positive cells. Ten representative high-power fields, selected randomly, were counted and each field counted was no less than 100 cells. Scores were determined by combining the proportion of positively stained carcinoma cells and the intensity of staining. The proportion of positively stained carcinoma cells was respectively scored as $1,<25 \%$ positive carcinoma cells; $2,25-50 \%$ positive carcinoma cells; and 3, >50\% positive carcinoma cells. The cells at each staining intensity were recorded on a scale of 0 , no staining; 1 , weak staining, light yellow; 2, moderate staining, yellowish brown; and 3, marked staining, brown. The staining index was calculated as follows: staining index $=$ staining intensity $\mathrm{x}$ proportion of positively stained carcinoma cells. Scores were attributed symbols as follows: 0, (-); 1-2, (+); 3-4, (++); and 6-9, (+++). Negative cells were defined as $(-)$ and positive cells were defined as $(+),(++)$ or $(+++)$.

Statistical analysis. Statistical analysis was performed using SPSS, version 15.0 software (SPPS Inc., Chicago, IL, USA). The difference between two groups was compared with a t-test. The $\chi^{2}$ test was used to measure the significance of the association between the NPC specimens and nasopharyngeal inflammation specimens. A four-fold table correlation was analyzed with Pearson's correlation analysis. The correlation of CDK3 expression with the clinical pathologic parameters of the patients was analyzed using the Wilcoxon test or Spearman's correlation test.

\section{Results}

CDK3 expression in NPC and nasopharyngeal epithelial cell lines. The expression pattern of CDK3 in cultured human NPC cell lines and the nasopharyngeal epithelial 

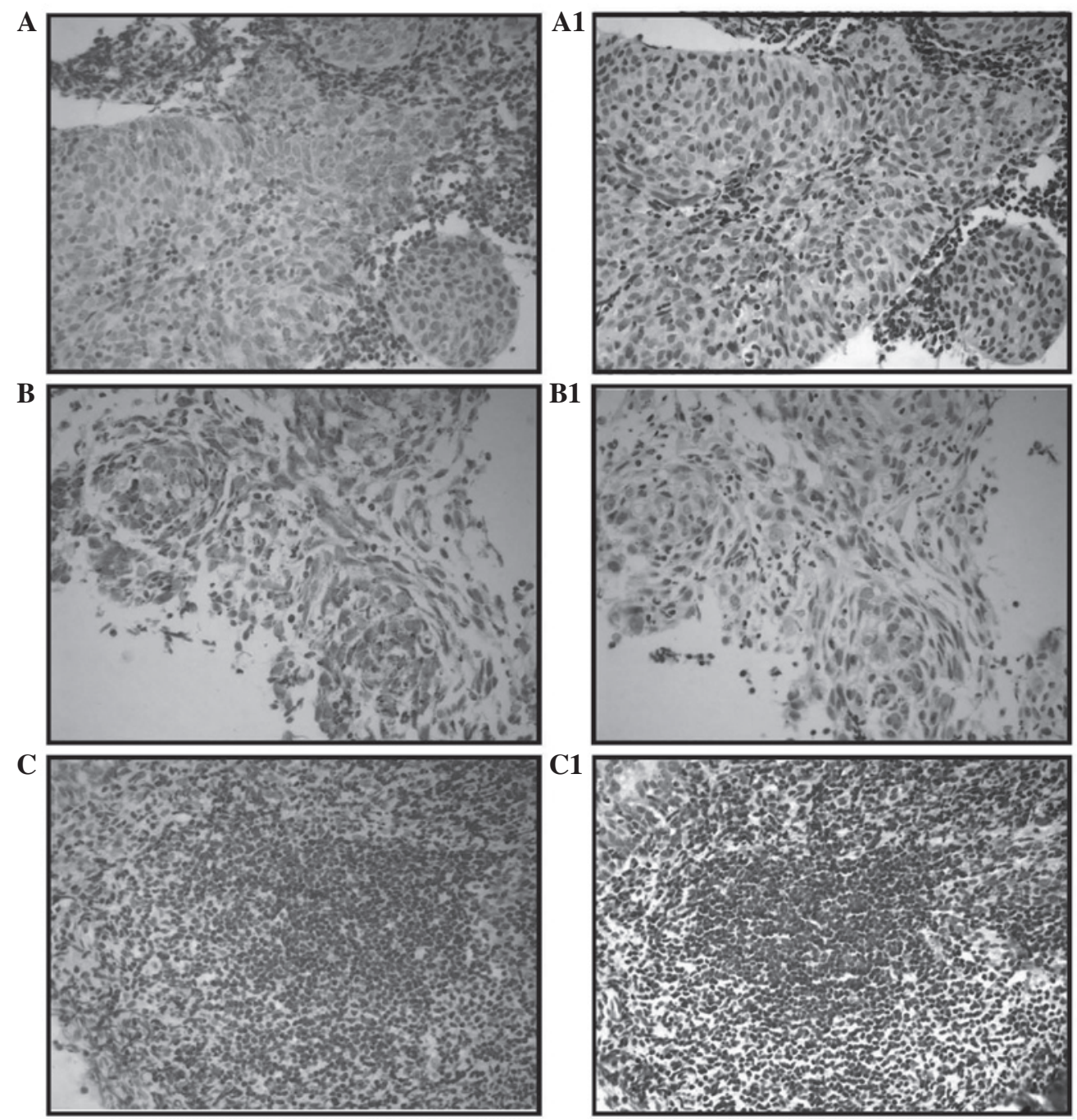

Figure 2. Immunohistochemical analysis of CDK3 protein expression in NPC tissue and inflamed tissue. (A-C) Protein immunohistochemistry (original magnification, x400); (A1-C1) the corresponding hematoxylin and eosin staining (original magnification, $\mathrm{x} 400$ ); (A and B) NPC specimens; (C) inflamed nasopharyngeal tissues. CDK3, cyclin-dependent kinase 3; NPC, nasopharyngeal carcinoma.

Table I. Expression of CDK3 protein in NPC and nasopharyngeal inflammation examined by IHC.

\begin{tabular}{lcccc}
\hline & & \multicolumn{2}{c}{$\mathrm{CDK} 3, \mathrm{n}(\%)$} & \\
\cline { 3 - 4 } Type & Total (n) & Positive & Negative & P-value \\
\hline NPC & 94 & $63(67.0)$ & $31(33.0)$ & \\
$\begin{array}{l}\text { Nasopharyngeal } \\
\text { inflammation }\end{array}$ & 40 & $5(12.5)$ & $35(87.5)$ & \\
Total & 134 & 68 & 66 & $<0.001$ \\
\hline
\end{tabular}

CDK3, cyclin-dependent kinase 3; NPC, nasopharyngeal carcinoma; IHC, immunohistochemistry.

cell line was investigated by western blotting. CDK3 protein expression was detectable in all cell lines. However, compared with the NP-69 nasopharyngeal epithelial cell line, CDK 3 was overexpressed in CNE1, CNE2 and 5-8F NPC cell lines (Fig. 1).
CDK3 expression in NPC and inflamed nasopharyngeal tissues by IHC staining. To determine whether the expression level of CDK3 protein is associated with the histological characteristics of NPC, CDK3 expression was examined in samples from 94 cases of NPC and 40 cases of nasopharyngeal inflammation by IHC staining. As shown in Fig. 2, CDK3 was found to be upregulated in NPC (Fig. 2A and B; NPC specimens) compared with in the tissues with nasopharyngeal inflammation (Fig. 2C). There were 63 cases that showed marked or moderate positive CDK3 staining in the 94 NPC samples $(67.0 \%)$ and only 5 cases out of the 40 cases with nasopharyngeal inflammation (12.5\%). The expression levels of CDK3 in NPC and tissues with nasopharyngeal inflammation were significantly different $(\mathrm{P}<0.001$; Table I).

Expression of CDK3 protein and clinical pathological parameters of NPC. To determine whether the expression level of CDK3 protein is associated with the clinical pathological parameters of NPC, 94 NPC clinical specimens, which included 18 cases of stage I, 51 cases of stage II, 18 cases of stage III and 7 cases of stage IV NPC, were examined by IHC staining with an antibody against human CDK3. No significant associations were 
Table II. Correlation between CDK3 expression and clinical pathological parameters analyzed by Spearman's correlation test.

\begin{tabular}{|c|c|c|c|c|c|c|}
\hline \multirow{2}{*}{$\begin{array}{l}\text { Clinical pathological } \\
\text { parameter }\end{array}$} & \multirow[b]{2}{*}{$\mathrm{n}$} & \multicolumn{4}{|c|}{ CDK3 expression } & \multirow[b]{2}{*}{ P-value } \\
\hline & & - & + & ++ & +++ & \\
\hline \multicolumn{7}{|l|}{ Degree of infiltration } \\
\hline $\mathrm{T} 1$ & 38 & 15 & 14 & 8 & 1 & \multirow[t]{4}{*}{$<0.001$} \\
\hline $\mathrm{T} 2$ & 40 & 13 & 8 & 11 & 8 & \\
\hline $\mathrm{T} 3$ & 13 & 3 & 1 & 4 & 5 & \\
\hline $\mathrm{T} 4$ & 3 & 0 & 0 & 0 & 3 & \\
\hline \multicolumn{7}{|l|}{ Lymph node metastasis } \\
\hline NO & 29 & 12 & 8 & 4 & 5 & \multirow[t]{4}{*}{$<0.001$} \\
\hline N1 & 52 & 16 & 14 & 14 & 8 & \\
\hline $\mathrm{N} 2$ & 9 & 2 & 1 & 3 & 3 & \\
\hline N3 & 4 & 1 & 0 & 2 & 1 & \\
\hline \multicolumn{7}{|l|}{ TNM clinical stage } \\
\hline $\mathrm{I}$ & 18 & 9 & 6 & 3 & 0 & \multirow[t]{4}{*}{$<0.001$} \\
\hline II & 51 & 17 & 15 & 12 & 7 & \\
\hline III & 18 & 4 & 2 & 6 & 6 & \\
\hline IV & 7 & 1 & 0 & 2 & 4 & \\
\hline
\end{tabular}

CDK3, cyclin-dependent kinase 3; TNM, tumor node metastasis.

observed between CDK3 expression and patient gender (data not shown). However, it was noted that there was a significant correlation between CDK3 protein expression and NPC tumor stage ( $\mathrm{T}$ stage) $(\mathrm{P}<0.001)$, regional lymph nodal metastasis ( $\mathrm{N}$ stage $)(\mathrm{P}<0.001)$ and clinical stage $(\mathrm{P}<0.001$; Table II $)$.

\section{Discussion}

Activation of CDKs induced by overexpression of activator cyclins has been observed in numerous types of human cancer (19). A number of studies have demonstrated that CDK3 was expressed in various human tissues and cell lines $(7,12,20)$. Our previous study also found that CDK3 was overexpressed in glioblastoma tissues and in numerous human cancer cell lines (15). In the present study, CDK3 expression was observed to be elevated significantly in NPC cell lines compared with the nasopharyngeal epithelial cell line, providing further evidence to support the hypothesis that CDK3 is important role NPC tumorigenesis.

CDK3 is an important protein in cell cycle regulation. CDK3 activity occurs early in the G1 phase (21), peaks at mid-G1 (22) and is required for entry into the S phase (13), resulting in increased proliferation, as well as cell transformation (22). This was confirmed in our previous study; for example, knockdown of CDK3 suppressed proliferation and colony formation of T98G glioblastoma cells in soft agar, and suppressed foci formation induced by RasG12V/CDK3/ATF1 in NIH3T3 cells (15). It has been reported that the occurrence of NPC was closely associated with the inactivation of cell cycle-dependence kinase inhibitors, including p16 and p27 (11,23-25) and the overexpression of G1 cyclin D1, G1 cyclin E1 and CDK4 (26,27), suggesting that abnormal expression of cell cycle-associated proteins and protein kinases was directly involved in cell proliferation and malignant transformation of NPC, and therefore was important in the occurrence and development of NPC. In the present study, the expression levels of CDK3 were significantly overexpressed in NPC tissues compared with non-tumor nasopharyngeal epithelium, indicating that CDK3 may be involved in the pathogenesis of NPC. Furthermore, CDK3 expression was correlated significantly with $\mathrm{T}$ and $\mathrm{N}$ classification and clinical stage, suggesting that the overexpression of CDK3 was associated with aggressive tumor behavior in patients with NPC.

Correct subcellular localization of proteins is critical for their function and for accurate activation of the appropriate pathways by providing physiological context. Aberrant localization of proteins contributes to a number of disorders and diseases, including metabolic, cardiovascular, neurodegenerative diseases and cancer (28). The precise activation of CDKs is mediated through association with a regulatory cyclin subunit, phosphorylation of CDK and subcellular localization (29). In a human prostate cancer cell line, vitamin D-mediated inhibition of cell proliferation was correlated with relocalization of CDK2 from nuclear to cytoplasmic compartments and a significant decrease in CDK2 activity $(30,31)$. Cytoplastic localization of CDK4/6 has functions in the differentiation of pluripotent embryonic cells (32). In the current study, the cytoplasm and nuclei were found to be attached to brown or yellow particles and the majority of the particles were located in the cytoplasm, indicating that CDK3 was primarily localized in the cytoplasm of NPC cells. This finding suggested the possibility that upregulated expression of cytoplasmic CDK3 may provide a selective advantage in the occurrence and progression of NPC. Thus, cytoplastic localization of CDK3 may be beneficial in the development of biomarkers for predicting the progression of NPC patients. 
In conclusion, CDK 3 expression was characterized in NPC cell lines and clinical tissue specimens by western blotting and IHC, respectively. The expression of CDK3 was elevated in NPC cell lines compared with that in the nasopharyngeal epithelial cell line, and the CDK3 positive expression rate in NPC specimens was significantly higher compared with the inflamed nasopharyngeal tissue specimens. Furthermore, the expression level of CDK3 was markedly correlated with the histological stage of NPC. These results revealed that CDK3 may be a prognostic biomarker in NPC patients. Further studies are required to verify these findings and to clarify the role of CDK3 in NPC.

\section{Acknowledgements}

This study were supported by grants from the National Science Foundation of China (grant nos. 81071655, 30871247, 81171921 and 81172282), Shenzhen Peacock Plan (KQCX20130621101141669) and the Science and Technology Bureau of Shenzhen city grant (grant nos. JC20 1006010727A,JCYJ20120613165853326, JCR201110056 and ZDSY20130329101130496).

\section{References}

1. Mutirangura A,Tanunyutthawongese C,Pornthanakasem W, et al: Genomic alterations in nasopharyngeal carcinoma: loss of heterozygosity and Epstein-Barr virus infection. Br J Cancer 76: 770-776, 1997

2. Wee JT, Ha TC, Loong SL and Qian CN: Is nasopharyngeal cancer really a 'Cantonese cancer'? Chin J Cancer 29: 517-526, 2010.

3. No authors listed: Cancer incidence in five continents. Volume VII. IARC Sci Publ: i-xxxiv, 1-1240, 1997.

4. Chow E, Payne D, O'Sullivan B, et al: Radiotherapy alone in patients with advanced nasopharyngeal cancer: comparison with an intergroup study. Is combined modality treatment really necessary? Radiother Oncol 63: 269-274, 2002.

5. al-Sarraf $M$ and McLaughlin PW: Nasopharynx carcinoma: choice of treatment. Int J Radiat Oncol Biol Phys 33: 761-763, 1995

6. Chua DT, Sham JS and Au GK: A phase II study of capecitabine in patients with recurrent and metastatic nasopharyngeal carcinoma pretreated with platinum-based chemotherapy. Oral Oncol 39: 361-366, 2003.

7. Meyerson M, Enders GH, Wu CL, et al: A family of human cdc2-related protein kinases. EMBO J 11: 2909-2917, 1992.

8. Miyata Y, Liu Y, Jankovic V, et al: Cyclin C regulates human hematopoietic stem/progenitor cell quiescence. Stem Cells 28: 308-317, 2010.

9. Rao HV, Thirumangalakudi L and Grammas P: Cyclin C and cyclin dependent kinases 1,2 and 3 in thrombin-induced neuronal cell cycle progression and apoptosis. Neurosci Lett 450: 347-350, 2009.

10. Sage J, Miller AL, Pérez-Mancera PA, Wysocki JM and Jacks T: Acute mutation of retinoblastoma gene function is sufficient for cell cycle re-entry. Nature 424: 223-228, 2003.

11. Hofmann F and Livingston DM: Differential effects of cdk2 and cdk3 on the control of $\mathrm{pRb}$ and E2F function during G1 exit. Genes Dev 10: 851-861, 1996.

12. Ren S and Rollins BJ: Cyclin C/cdk3 promotes Rb-dependent G0 exit. Cell 117: 239-251, 2004
13. van den Heuvel S and Harlow E: Distinct roles for cyclin-dependent kinases in cell cycle control. Science 262: 2050-2054, 1993.

14. Lee JY, Jeong W, Kim JH, et al: Distinct expression pattern and post-transcriptional regulation of cell cycle genes in the glandular epithelia of avian ovarian carcinomas. PLoS One 7: e51592, 2012

15. Zheng D, Cho YY, Lau AT, et al: Cyclin-dependent kinase 3-mediated activating transcription factor 1 phosphorylation enhances cell transformation. Cancer Res 68: 7650-7660, 2008.

16. Cho YY,Tang F, YaoK, et al: Cyclin-dependent kinase-3-mediated c-Jun phosphorylation at Ser63 and Ser73 enhances cell transformation. Cancer Res 69: 272-281, 2009.

17. Bullrich F, MacLachlan TK, Sang N, et al: Chromosomal mapping of members of the cdc2 family of protein kinases, cdk3, cdk6, PISSLRE, and PITALRE, and a cdk inhibitor, p27Kip1, to regions involved in human cancer. Cancer Res 55: 1199-1205, 1995.

18. Mao YP, Li WF, Chen L, et al: A clinical verification of the Chinese 2008 staging system for nasopharyngeal carcinoma. Ai Zheng 28: 1022-1028, 2009 (In Chinese).

19. Malumbres M and Barbacid M: Mammalian cyclin-dependent kinases. Trends Biochem Sci 30: 630-641, 2005.

20. Schang LM, Bantly A and Schaffer PA: Explant-induced reactivation of herpes simplex virus occurs in neurons expressing nuclear cdk2 and cdk4. J Virol 76: 7724-7735, 2002.

21. Keezer SM and Gilbert DM: Evidence for a pre-restriction point Cdk3 activity. J Cell Biochem 85: 545-552, 2002.

22. Braun K, Hölzl G, Soucek T, Geisen C, Möröy T and Hengstschläger M: Investigation of the cell cycle regulation of cdk3-associated kinase activity and the role of cdk3 in proliferation and transformation. Oncogene 17: 2259-2269, 1998.

23. Alessandrini A, Chiaur DS and Pagano M: Regulation of the cyclin-dependent kinase inhibitor p27 by degradation and phosphorylation. Leukemia 11: 342-345, 1997.

24. Baba Y, Tsukuda M, Mochimatsu I, et al: Reduced expression of p16 and p27 proteins in nasopharyngeal carcinoma. Cancer Detect Prev 25: 414-419, 2001.

25. Pan Y, Zhang Q, Tian L, et al: Jab1/CSN5 negatively regulates p27 and plays a role in the pathogenesis of nasopharyngeal carcinoma. Cancer Res 72: 1890-1900, 2012

26. Shih LC, Tsai CW, Tsai MH, et al: Association of cyclin D1 genotypes with nasopharyngeal carcinoma risk. Anticancer Res 32: 1093-1098, 2012.

27. Acikalin MF, Etiz D, Gurbuz MK, Ozudogru E, Canaz F and Colak E: Prognostic significance of galectin-3 and cyclin D1 expression in undifferentiated nasopharyngeal carcinoma. Med Oncol 29: 742-749, 2012.

28. Hung MC and Link W: Protein localization in disease and therapy. J Cell Sci 124: 3381-3392, 2011.

29. Shapiro GI: Cyclin-dependent kinase pathways as targets for cancer treatment. J Clin Oncol 24: 1770-1783, 2006.

30. Yang ES and Burnstein KL: Vitamin D inhibits G1 to $S$ progression in $\mathrm{LNCaP}$ prostate cancer cells through $\mathrm{p} 27 \mathrm{Kip} 1$ stabilization and Cdk2 mislocalization to the cytoplasm. J Biol Chem 278: 46862-46868, 2003.

31. Flores O, Wang Z, Knudsen KE and Burnstein KL: Nuclear targeting of cyclin-dependent kinase 2 reveals essential roles of cyclin-dependent kinase 2 localization and cyclin $\mathrm{E}$ in vitamin D-mediated growth inhibition. Endocrinology 151: 896-908, 2010.

32. Bryja V,Pacherník J, Vondrácek J, et al: Lineage specific composition of cyclin D-CDK4/CDK6-p27 complexes reveals distinct functions of CDK4, CDK6 and individual D-type cyclins in differentiating cells of embryonic origin. Cell Prolif 41: 875-893, 2008. 